\title{
Vojta Approach Affects Neck Stability and Static Balance in Sitting Position of Children With Hypotonia
}

\author{
Sun-Young $\mathrm{Ha}^{1}$, Yun-Hee Sung ${ }^{1,2}$ \\ ${ }^{1}$ Department of Physical Therapy, Graduate School, Kyungnam University, Changwon, Korea \\ ${ }^{2}$ Department of Physical Therapy, College of Health Sciences, Kyungnam University, Changwon, Korea
}

Purpose: In this study, the effect of the Vojta approach on neck stability and static balance in children with hypotonia was studied.

Methods: Seventeen children with hypotonia were randomly divided into the Vojta approach group $(n=9)$ and the general physical therapy group $(n=8)$. Each group was applied intervention for 30 minutes per session, 3 times a week, for a total of 4 weeks. Ultrasonography was used to measure deep neck flexor muscle thickness, craniovertebral angle (CVA) to measure neck alignment along the spine segment, and Balancia software program to measure static balance.

Results: In the Vojta approach group, the deep neck flexor muscle thickness was significantly increased $(\mathrm{P}<0.05)$, and the CVA was significantly improved $(\mathrm{P}<0.05)$. In addition, path area among static balance was significantly improved $(\mathrm{P}<0.05)$. Conclusions: The Vojta approach can be suggested as an effective intervention method for improving neck stability and static balance in children with hypotonia.

Keywords: Deep neck flexor muscle; Craniovertebral angle; Vojta approach; Static balance; Hypotonia

- Research Ethics: This study was conducted after approval by the Research Ethics Committee of Kyungnam University (1040460-A-2020-016).

- Conflict of Interest: No potential conflict of interest relevant to this article was reported.

\footnotetext{
- HIGHLIGHTS

- Children with hypotonia reduced gross motor function.

- Low muscle tone affected the posture and reduces the balance in children with hypotonia

- The Vojta approach affected postural improvement by activating the deep spinal muscles.
}

\section{INTRODUCTION}

The Vojta approach is an intervention method that elicits a systemic motor response by stimulating a specific reflex zone in a specific starting position [1]. Stimulating the reflex zone of the Vojta approach inhibits abnormal movements and induces normal motor development by repeatedly remembering normal movements in the brain [2]. In addition, since it has a certain direction of muscle contraction, it stimulates muscles, joints, ligaments, and tendons, and postural maintenance can be improved through isometric contraction [3]. Based on this purpose, the Vojta approach has been applied to various diseases such as children with developmental delay (DD) [1], stroke patients [4], and musculoskeletal diseases [5].

Children who present with weakness, low muscle tone, abnormal reflexes, and sensory and motor problems have devel-

Corresponding author: Yun-Hee Sung (i) https://orcid.org/0000-0002-4877-9784 Department of Physical Therapy, College of Health Sciences, Kyungnam University, 7 Kyungnamdaehak-ro, Masanhappo-gu, Changwon 51767, Korea Email: sungpt97@kyungnam.ac.kr

Submitted: September 2, 2021 / Accepted after revision: October 22, 2021 
opmental delays and are considered hypotonia [6]. They are sometimes accompanied with seizures and organ malformation $[7,8]$. Many disorders, including neurological disorders, endocrine and metabolic disorders, Prader-Willi syndrome, and spinal muscular atrophy, often present with hypotonia as a clinical symptom [6]. Hypotonia is characterized by lack of resistance to passive movements, joint hypermobility, and reduced endurance $[8,9]$. This causes abnormalities in the posture of tilting the head back in a sitting position, and excessive wrinkles appear on the neck, which lowers the stability of the neck $[10,11]$.

Neck stability refers to the action of the deep neck flexor muscle, the antigravity flexor muscle that controls the head movement [11]. The deep neck flexor muscles have a positive effect on the correct alignment of the neck and the balance of the trunk and neck and play a role in providing neck stability during movement $[12,13]$. They are attached directly to the cervical spine and joints and play an important role in the postural control of the cervical spine and each segment stability [14]. Weak deep neck flexor muscle can lead to cervical spine instability [12]. This problem causes the forward head posture (FHP), which affects balance due to incorrect afferent input from the neck muscles [15].

Balance is the basis of motor development, and tonic activation of the trunk muscles is necessary to maintain an upright sitting posture $[16,17]$. Hypotonia are difficult to maintain balance due to low muscle tone, and postural sway are greater than those of typical developing children [18]. Abnormal posture is a major factor that reduces balance ability and quality of life $[19,20]$, and balance disorders affect daily life by limiting interactions with the environment [21]. Therefore, it is important to improve balance in children with hypotonia.

Various interventions such as treadmill, dual task, neck and trunk stabilization exercise, and Vojta approach have been applied to improve motor function $[1,9,15,22,23]$. Among them, the Vojta approach has been reported to influence postural improvement by activating the deep spinal muscles [3]. Studies on the Vojta approach mainly focuses on children with hypertonia, and there are few studies on children with hypotonia. In addition, the neck stability and balance in children with hypotonia are decreased, but studies on this ae rare. Therefore, we determined the effect of the Vojta approach on neck stability and static balance in sitting position in children with hypotonia.

\section{MATERIALS AND METHODS}

\section{Participants}

The experiment was performed on children with hypotonia who were admitted to hospital. Th inclusion criteria were as follows: (1) children diagnosed with DD before the age of 5 and accompanied by hypotonia, (2) gross motor function classification system level II-IV, (3) children who can perform simple verbal instructions from the researcher, (4) children who can maintain a sitting posture. The exclusion criteria were as follows: (1) children with DD accompanied by spasticity, (2) children with vision and hearing impairments, (3) children with uncontrolled seizure, (4) children with acute fever or inflammation. All participants were informed of the study and signed consent forms. This study was conducted after approval by the Research Ethics Committee of the Kyungnam University (1040460-A-2020-016).

Participants were divided into 2 groups (experimental group and control group). The general characteristics of the participants were as follows: experimental group $(n=9)$, age $46.88 \pm$ 20.39 months, gross motor function classification system (GMFCS) $\mathrm{I} / \mathrm{II} / \mathrm{III} / \mathrm{IV}=1 / 2 / 4 / 2$ and control group $(\mathrm{n}=8)$, age $54.87 \pm$ 19.09 months, GMFCS I/II/III/IV =1/2/3/2. The experimental group applied the Vojta approach, and the control group applied general physical therapy. Intervention was applied to each group for 30 minutes per session, twice a day, 3 times a week, for a total of 4 weeks. All groups were examined for deep neck flexor muscle thickness, craniovertebral angle (CVA), static balance before and after intervention.

\section{Intervention}

The Vojta approach is divided into reflex turning 1, reflex turning 2, reflex creeping, and first position. The reflex turning 1 stimulated the breast zone in the supine position, and reflex turning 2 stimulated the medial border of the scapula and the anterior superior iliac spine (ASIS) in the side lying position. Reflex creeping stimulated the medial epicondyle of the humerus and calcaneus in the prone position. In the first position, both knees bent in the prone position, and the upper limbs were in the same position as the reflex creeping, stimulating the ASIS and the medial epicondyle of the humerus. Each posture was applied for a total of 30 minutes, 10 minutes each, and reflex creeping or the first position was selected according to the characteristics of the child. 


\section{Ultrasonography}

The thicknesses of deep neck flexor muscle were scanned images in B mode using SONON application, and a $10-\mathrm{MHz}$ linear probe of ultrasonography (Sonson, Healcerion, Seoul, Korea). Participants were seated with their feet on the floor, with the spine extended and maintained as far as possible. The probe was placed vertically at a position $3 \mathrm{~cm}$ outward from the center of the trachea to measure the thickness of the right deep neck flexor muscle [24]. It was measured at the end of the exhalation to reduce the error due to respiration. For the thickness of the muscle, a baseline was determined between the cervical spine 4 and 5, and then 3 lines were drawn at $0.5 \mathrm{~cm}$ intervals to measure the length, and the average value of 6 lines for $2 \mathrm{im}$ ages was used. The deep neck flexor muscle thickness was measured by NIH Image J ver. 1.47 (Sun Microsystems Inc., Stapleton, NY, USA) (Fig. 1).

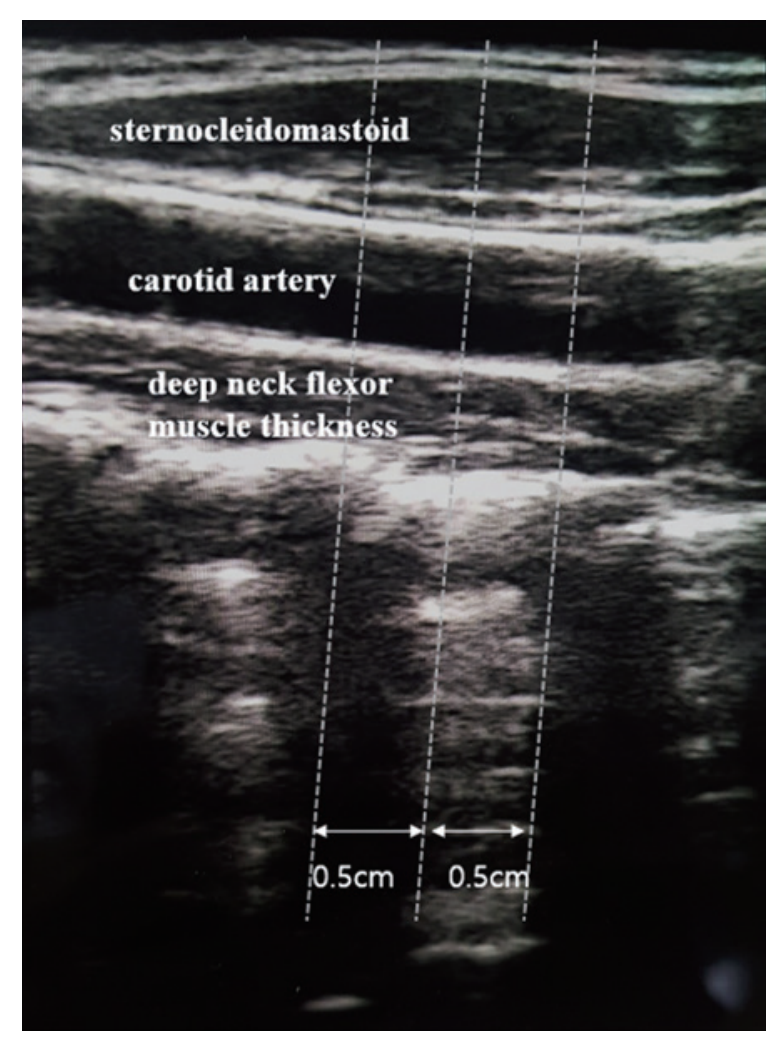

Fig. 1. Ultrasonography imaging of the deep neck flexor muscle in children with hypotonia. The deep neck flexor muscle thickness was measured in a sitting position with the back as straight as possible. A baseline was established between cervical vertebra 4 and 5 , and 3 lines were drawn at $0.5-\mathrm{cm}$ intervals to measure the length.

\section{Craniovertebral Angle}

To measure CVA, the examiner asked the child to remain in a sitting position and stare straight ahead. We installed and recorded Galaxy Tab S3 (Samsung, Seoul, Korea) at 3-m distance and a $0.7-\mathrm{m}$ height on the right side of the child to measure the CVA according to each segment. CVA was measured when supporting 5 segments of the spine: thoracic spine 3 (T3), T7, T11, lumbar spine 3 (L3), sacral spine (S1). Eight small round stickers $(2 \mathrm{~cm} \times 2 \mathrm{~cm} \times 2 \mathrm{~cm})$ were attached to C7, T3, T7, T11, L3, S1, ear tragus, temporal lobe sulcus to accurately track the vertebral segment and CVA. CVA measured the angle formed by the horizontal line passing through the spinous process of $\mathrm{C} 7$ and the line connecting C7 and ear tragus [25]. The angle was measured according to the segment using the Dartfish software program (Dartfish 7, Lausanne, Switzerland). The reliability for the CVA is 0.88 [25].

\section{Static Balance}

To measure the static balance in the sitting position, center of pressure information for the $\mathrm{X}$ and $\mathrm{Y}$ axes was collected using a Wii Balance Board (Nintendo, Kyoto, Japan). The collected data were measured for path length, path velocity and path area using Balancia software version 2.0 (Minto Systems, Seoul, Korea). Participants were seated on a Wii balance board at the height of the feet not touching the floor, with their arms relaxed and gazing straight ahead. They kept their eyes open for $20 \mathrm{sec}-$ onds and were measured twice and the average value was used.

\section{Date Analysis}

IBM SPSS version 21.0 (IBM Corp., Armonk, NY, USA) was used for statistical analysis. Shapiro-Wilks test was performed to test the normality of variables. We used the independent $t$ test for differences between groups and the paired $t$-test for difference within group. All levels of statistical significance $(\alpha)$ were set to 0.05 .

\section{RESULTS}

\section{Comparison of Deep Neck Flexor Muscle Thickness}

In the experimental and control group, there was a significant difference within group $(\mathrm{P}<0.05)$ (Table 1$)$.

\section{Comparison of CVA}

T7, T11, L3, and S1 were significantly different between the groups in postintervention $(\mathrm{P}<0.05)$. In the experimental 
Table 1. Comparison of deep neck flexor muscle thickness $(\mathrm{cm})$

\begin{tabular}{|c|c|c|c|c|c|}
\hline \multicolumn{3}{|c|}{ Experimental } & \multicolumn{3}{|c|}{ Control } \\
\hline Pre & Post & Post-pre & Pre & Post & Post-pre \\
\hline $0.45 \pm 0.10^{\dagger}$ & $0.54 \pm 0.50^{*}$ & $0.09 \pm 0.11$ & $0.53 \pm 0.10$ & $0.56 \pm 0.06^{*}$ & $0.03 \pm 0.06$ \\
\hline
\end{tabular}

Values are presented as mean \pm standard deviation.

${ }^{\star} \mathrm{P}<0.05$, significant difference within group. ${ }^{\dagger} \mathrm{P}<0.05$, significant difference from control.

Table 2. Comparison of CVA in sitting position $\left({ }^{\circ}\right)$

\begin{tabular}{|c|c|c|c|c|c|c|}
\hline \multirow{2}{*}{ Variable } & \multicolumn{3}{|c|}{ Experimental } & \multicolumn{3}{|c|}{ Control } \\
\hline & Pre & Post & Post-pre & Pre & Post & Post-pre \\
\hline $\mathrm{T} 3$ & $36.57 \pm 8.66$ & $37.57 \pm 9.23$ & $1.00 \pm 8.66$ & $34.28 \pm 5.85$ & $35.03 \pm 5.40$ & $0.74 \pm 4.76$ \\
\hline T7 & $39.30 \pm 7.27$ & $43.77 \pm 3.78^{\star, \dagger}$ & $4.47 \pm 5.81$ & $37.07 \pm 3.99$ & $39.26 \pm 5.12^{*}$ & $2.18 \pm 2.07$ \\
\hline $\mathrm{T} 11$ & $42.26 \pm 4.11$ & $46.68 \pm 5.81^{\star, \dagger}$ & $4.42 \pm 3.06^{\dagger}$ & $40.45 \pm 5.09$ & $40.17 \pm 4.83$ & $-0.28 \pm 3.18$ \\
\hline L3 & $39.98 \pm 5.45$ & $43.57 \pm 4.87^{\star, \dagger}$ & $3.45 \pm 9.42^{\dagger}$ & $40.04 \pm 3.67$ & $37.96 \pm 5.66$ & $-2.18 \pm 6.49$ \\
\hline S1 & $38.63 \pm 4.54$ & $42.27 \pm 5.51^{*, \dagger}$ & $3.63 \pm 4.66^{\dagger}$ & $37.12 \pm 2.35$ & $36.83 \pm 5.30$ & $-0.28 \pm 6.18$ \\
\hline
\end{tabular}

Values are presented as mean \pm standard deviation.

CVA, craniovertebral angle; T3, thoracic spine 3; T7, thoracic spine 7; T11, thoracic spine 11; L3, lumbar spine 3; S1, sacral spine 1.

${ }^{\star} \mathrm{P}<0.05$, significant difference within group. ${ }^{\dagger} \mathrm{P}<0.05$, significant difference from control.

Table 3. Comparison of static balance in sitting position

\begin{tabular}{lcccccccc}
\hline \multirow{2}{*}{ Variable } & \multicolumn{3}{c}{ Experimental } & & & \multicolumn{3}{c}{ Control } \\
\cline { 2 - 3 } & Pre & Post & & Post-pre & & Pre & Post & Post-pre \\
\hline Path velocity $(\mathrm{cm} / \mathrm{sec})$ & $12.98 \pm 5.52$ & $11.87 \pm 3.31$ & & $-1.10 \pm 2.40$ & & $11.07 \pm 5.62$ & $10.13 \pm 3.66$ & $-0.93 \pm 2.52$ \\
Path length $(\mathrm{cm})$ & $25.08 \pm 10.12$ & $24.32 \pm 7.31$ & & $-0.75 \pm 3.62$ & & $22.60 \pm 7.27$ & $21.19 \pm 6.75$ & $-1.21 \pm 7.34$ \\
Path area $\left(\mathrm{cm}^{2}\right)$ & $12.25 \pm 5.16$ & $8.57 \pm 2.17^{\star}$ & & $-3.67 \pm 4.91$ & & $12.07 \pm 4.82$ & $9.44 \pm 4.13^{*}$ & $-2.63 \pm 4.00$ \\
\hline
\end{tabular}

Values are presented as mean \pm standard deviation.

${ }^{\star} \mathrm{P}<0.05$, significant difference within group.

group, there was a significant difference within the group in T7, T11, L3, and S1 $(\mathrm{P}<0.05)$. The change (post-pre) in T11, L3, and $\mathrm{S} 1$ was significantly different between the groups $(\mathrm{P}<0.05)$ (Table 2).

\section{Comparison of Static Balance in Sitting Position}

In the experimental and control group, there was a significant difference within group $(\mathrm{P}<0.05)$ (Table 3$)$.

\section{DISCUSSION}

Children with hypotonia show delayed motor development [6]. The Vojta approach allows the body to be erected and postural control through tonic muscle contraction of the neck, trunk, and limbs [4].

In this study, the thickness of the deep neck flexor muscle and the CVA were increased in the Vojta approach group. Low muscle tone of the trunk in children with hypotonia increases the bending of the lumbar spine in the sitting position and affects the kyphosis of the thoracic spine [6]. This leads to postural misalignment, which causes FHP and ultimately reduces quality of life $[9,19]$. The FHP reduces the CVA in the sitting position and causes deep neck flexor muscle weakness [26]. The deep neck flexor muscle is interconnected with the deep muscles of the spine located below the cervical spine, and the instability of the vertebral segment eventually affects the instability of the cervical spine [27]. Ishida et al. [26] reported a correlation between deep neck flexor muscle and CVA in healthy people, as the FHP does not use the deep neck flexor muscle in a sitting position, resulting in a smaller thickness of the deep neck flexor muscle. Falla et al. [14] reported that craniocervical training improved the upright posture of the cervical spine 
when craniocervical training and endurance strength training of deep neck flexion muscles were applied to patients with chronic neck pain. This is a result of a direct improvement of the deep neck flexors of the cervical spine, and inadequate control of the head was functionally related to deep cervical muscle damage. Kang et al. [28] found that when scapular stabilization exercise was applied to FHP patients, SCM activity decreased and CVA increased. This increased the activation of muscles scapular around the scapula, which affected the entire upper body and improved the round shoulder and spine alignment. Previous studies show that when the deep neck flexor muscle thickens, CVA increases, and the posture of the spine also affects neck alignment. In this study, it is thought that deep neck flexor muscle stimulation and spinal extension through reflex creeping of the Vojta approach activated deep spinal muscles. In addition, it is thought that the increase in the thickness of the deep neck flexor muscle improved the CVA by increasing the stability of the spine. The CVA was improved when supporting all vertebral segments except $\mathrm{T} 3$ in the experimental group, and these results suggest that stimulation of the deep spinal muscles also affects the posture of the cervical spine.

The misalignment of the body makes it difficult to control the head and trunk in a sitting or standing position and causes problems with balance and postural control [29]. Children with hypotonia have decreased flexibility due to hypotonia and lack of motivation, resulting in increased postural sway than typical developmental children [30]. Therefore, reduction of postural sway in children with hypotonia can be the goal of intervention. Shin et al. [15] reported that the static balance improved when a neck and trunk stabilization exercise was applied to children with spastic CP. This is a result of the improvement of trunk and neck muscle strength, they mentioned that the balance was improved by integrating sensory information in the central nervous system to perform movement. Park et al. [31] reported that when postural control training in a sitting position using virtual reality for children with spasticity, balance ability improved in the sitting position. In this study, path area among the static balance was improved in the Vojta approach group. These results suggest that the tonic muscle contraction through the Vojta approach improved afferent information of the neck, affecting neck stability and static balance. However, the path velocity and path length were not significantly different from those of the general physical therapy group, which is thought to be due to the role of visual information monitoring the body position when looking straight ahead to maintain a sitting pos- ture and the short duration of intervention.

Summarizing the results of this study, the vertebral muscles strengthened through the Vojta approach influenced the increase in the thickness of the deep neck flexors, thereby improving the stability of the neck, which increased CVA. In addition, the improvement of afferent information through neck stability influenced the static balance. Therefore, the Vojta approach can be suggested as an effective intervention for improving neck stability and balance in children with hypotonia. The limitation of this study is that it is difficult to generalize the contents because the number of participants is small and the intervention period is short. In addition to experimental interventions, participants had difficulty controlling variables in their daily lives. Therefore, future research should be conducted to supplement these limitations.

\section{AUTHOR CONTRIBUTION STATEMENT}

- Conceptualization: $S Y H$

- Data curation: $S Y H$

- Formal analysis: $S Y H$

- Funding acquisition: YHS

- Methodology: SYH

- Project administration: YHS

- Visualization: YHS

-Writing-original draft: YHS

- Writing-review \& editing: YHS

\section{ORCID}

$\begin{array}{ll}\text { Sun-Young Ha } & 0000-0001-7715-9579 \\ \text { Yun-Hee Sung } & 0000-0002-4877-9784\end{array}$

\section{REFERENCES}

1. Sung YH, Ha SY. The Vojta approach changes thicknesses of abdominal muscles and gait in children with spastic cerebral palsy: a randomized controlled trial, pilot study. Technol Health Care 2020; 28:293-301.

2. Khan MH, Helsper J, Farid MS, Grzegorzek M. A computer visionbased system for monitoring Vojta therapy. Int J Med Inform 2018; 113:85-95.

3. Ha SY, Sung YH. Effects of Vojta method on trunk stability in healthy individuals. J Exerc Rehabil 2016;12:542-7.

4. Epple C, Maurer-Burkhard B, Lichti MC, Steiner T. Vojta therapy 
improves postural control in very early stroke rehabilitation: a randomised controlled pilot trial. Neurol Res Pract 2020;2:23.

5. Jung MW, Landenberger M, Jung T, Lindenthal T, Philippi H. Vojta therapy and neurodevelopmental treatment in children with infantile postural asymmetry: a randomised controlled trial. J Phys Ther Sci 2017;29:301-6.

6. Martin K, Kaltenmark T, Lewallen A, Smith C, Yoshida A. Clinical characteristics of hypotonia: a survey of pediatric physical and occupational therapists. Pediatr Phys Ther 2007;19:217-26.

7. Moon JU, Cho KO. Current pharmacologic strategies for treatment of intractable epilepsy in children. Int Neurourol J 2021;25(Suppl 1):S8-18.

8. Kaler J, Hussain A, Patel S, Majhi S. Neuromuscular junction disorders and floppy infant syndrome: a comprehensive review. Cureus 2020;12:e6922.

9. Igarashi M. Floppy infant syndrome. J Clin Neuromuscul Dis 2004; 6:69-90.

10. Peredo DE, Hannibal MC. The floppy infant: evaluation of hypotonia. Pediatr Rev 2009;30:66-76.

11. Olson LE, Millar AL, Dunker J, Hicks J, Glanz D. Reliability of a clinical test for deep cervical flexor endurance. J Manipulative Physiol Ther 2006;29:134-8.

12. Sung YH. Upper cervical spine dysfunction and dizziness. J Exerc Rehabil 2020;16:385-91.

13. Ha SY, Sung YH. A temporary forward head posture decreases function of cervical proprioception. J Exerc Rehabil 2020;16:16874.

14. Falla D, Jull G, Russell T, Vicenzino B, Hodges P. Effect of neck exercise on sitting posture in patients with chronic neck pain. Phys Ther 2007;87:408-17.

15. Shin JW, Song GB, Ko J. The effects of neck and trunk stabilization exercises on cerebral palsy children's static and dynamic trunk balance: case series. J Phys Ther Sci 2017;29:771-4.

16. Lee NY, Lee EJ, Kwon HY. The effects of dual-task training on balance and gross motor function in children with spastic diplegia. J Exerc Rehabil 2021;17:21-7.

17. Masani K, Sin VW, Vette AH, Thrasher TA, Kawashima N, Morris $\mathrm{A}$, et al. Postural reactions of the trunk muscles to multi-directional perturbations in sitting. Clin Biomech (Bristol, Avon) 2009;24:17682.

18. Kyvelidou A, Harbourne RT, Stergiou N. Severity and characteristics of developmental delay can be assessed using variability measures of sitting posture. Pediatr Phys Ther 2010;22:259-66.

19. Bayattork M, Sköld MB, Sundstrup E, Andersen LL. Exercise inter- ventions to improve postural malalignments in head, neck, and trunk among adolescents, adults, and older people: systematic review of randomized controlled trials. J Exerc Rehabil 2020;16:3648.

20. Ha SY, Sung YH. The effect of scapular strengthening exercise using elastic band on balance and quality of life in the old people. J Exerc Rehabil 2021;17:214-9.

21. Ardolino E, Flores M, Manella K. Gross motor outcomes after dynamic weight-bearing in 2 children with trunk hypotonia: a case series. Pediatr Phys Ther 2017;29:360-4.

22. Hong M, Kim M, Kim TW, Park SS, Kim MK, Park YH, et al. Treadmill exercise improves motor function and short-term memory by enhancing synaptic plasticity and neurogenesis in photothrombotic stroke mice. Int Neurourol J 2020;24(Suppl 1):S28-38.

23. Ulrich DA, Ulrich BD, Angulo-Kinzler RM, Yun J. Treadmill training of infants with Down syndrome: evidence-based developmental outcomes. Pediatrics 2001;108:E84.

24. Jesus FM, Ferreira PH, Ferreira ML. Ultrasonographic measurement of neck muscle recruitment: a preliminary investigation. J Man Manip Ther 2008;16:89-92.

25. Fernández-de-las-Peñas C, Alonso-Blanco C, Cuadrado ML, Gerwin RD, Pareja JA. Trigger points in the suboccipital muscles and forward head posture in tension-type headache. Headache 2006;46: 454-60.

26. Ishida H, Suehiro T, Kurozumi C, Ono K, Ando S, Watanabe S. Correlation between neck slope angle and deep cervical flexor muscle thickness in healthy participants. J Bodyw Mov Ther 2015;19: 717-21.

27. Frank C, Kobesova A, Kolar P. Dynamic neuromuscular stabilization \& sports rehabilitation. Int J Sports Phys Ther 2013;8:62-73.

28. Kang JI, Choi HH, Jeong DK, Choi H, Moon YJ, Park JS. Effect of scapular stabilization exercise on neck alignment and muscle activity in patients with forward head posture. J Phys Ther Sci 2018;30: 804-8.

29. Duarte Nde A, Grecco LA, Franco RC, Zanon N, Oliveira CS. Correlation between pediatric balance scale and functional test in children with cerebral palsy. J Phys Ther Sci 2014;26:849-53.

30. Kyvelidou A, Harbourne RT, Willett SL, Stergiou N. Sitting postural control in infants with typical development, motor delay, or cerebral palsy. Pediatr Phys Ther 2013;25:46-51.

31. Park SH, Son SM, Choi JY. Effect of posture control training using virtual reality program on sitting balance and trunk stability in children with cerebral palsy. NeuroRehabilitation 2021;48:247-54. 